\title{
The Free Volume of Condensed Phases Confined in a Nanopore as Seen by Computer Simulations and Compared to PALS
}

\author{
D. RAČKO* \\ Polymer Institute, Slovak Academy of Sciences, Dúbravská cesta 9, 84541 Bratislava, Slovak Republic
}

\begin{abstract}
In this contribution the free volume of glycerol phase confined in a nanopore in a wide temperature range is computed. The computed free volume is compared to the previously computed values in the glycerol bulk. The mean cavity volumes are also discussed with the experimental measurements by positronium annihilation lifetime spectroscopy. The computer simulations show that in the case of the confined glycerol phase the mean cavity volumes are larger than in the simulated bulk, and also the temperature dependence has a different qualitative behavior; the computed data are in agreement with experimental measurements performed for glycerol in a pore of comparable size with diameter $6 \mathrm{~nm}$. The simulations also indicate that an aspect of filling a pore is important for experimentalists. In the case of a perfectly sealed pore the cavity volume is observed to rise with decreasing temperature.
\end{abstract}

DOI: $10.12693 /$ APhysPolA.125.785

PACS: 78.70.Bj, 61.20.Ja, 76.60.-k, 64.70.pm

\section{Introduction}

Confined systems represent a recent and actual topic in the physics of condensed matter. The confinement affects cooperative properties of molecules, such as $T_{\mathrm{g}}$ [1]. The positronium lifetime annihilation spectroscopy (PALS) measurements have shown further deviations observed in confined condensed phases as compared to the classical bulk system [2]. In this case, the measured free volume in the glycerol confined phase was increasingly higher towards lower temperatures when compared to the classical bulk system, reaching nearly twice the value at the lowest temperature in glass phase.

In the previous work, glycerol bulk in the super-cooled state was simulated [3]. A model to compute free volume microstructure was developed, too. The model was shown to successfully simulate free volume cavities with equivalent volumes as observed in the PALS experiment, and as such provides a realistic picture on the free volume structure $[3,4]$. In the present contribution the existing computational model is further used to acquire a picture on the free volume microstructure in glycerol condensed phase confined in an infinite cylindrical nanopore.

The size (radius) of the nanopore is $6 \mathrm{~nm}$, as in the real experiment for which the measurements on confined glycerol were conducted. The pore was carved into a structure of realistic CPG glass [5]. The structure of the pore as well as the glycerol phase was modeled by a fully-atomistic molecular mechanic potential. The structure was refined by molecular dynamics (MD) simulations.

The structures at different temperatures were prepared by two different ways. (i) A cylinder was carved from the previously obtained glycerol bulk structure at $300 \mathrm{~K}$

*e-mail: dusan.racko@savba.sk (liquid) [3], with a diameter of the nanopore, $6 \mathrm{~nm}$. This cylinder was inserted into the pore and MD cooling down started. This situation represents a perfectly sealed pore, with no glycerol molecules flowing in the pore as the temperature drops. The resulting structure in glass showed layered structure of glycerol molecules, with a large cavity in the center of the pore. As a result the mean cavity volume was larger in glass than in liquid. (ii) In the second approach the cylinders were made from appropriate bulk structures simulated at given temperature [3] and they were inserted into the pores. Structures were equilibrated by MD simulations runs. The computed cavity volume showed increasing trend with the temperature, however quantitatively different from that observed in the bulk. As seen from the simulations this was partially an effect of the vacancies in the CPG glass matrix, which increase the mean volume of cavities in glass and decrease the mean cavity volume in liquid. The structure observed in the experiment [2] most likely fell in between these two model cases and the mean cavity volume is given by a complex relationship between sorption and layering of glycerol molecules, as well as properties of silica matrix.

\section{Computer simulations methods}

\subsection{Simulation of amorphous silica}

In the amorphous silica structure the $-\mathrm{SiO}-$ groups create a continuous and chemically bonded random network with amorphous structure. This is obtained from the structure of a perfect $\mathrm{SiO}$ crystal by random displacing $\mathrm{SiO}$ groups according to the algorithm developed by Vink et al. [6]. In this algorithm the computed structure factor of the simulated structure is fitted to the experimental one by a reverse Monte Carlo algorithm until a perfect agreement is obtained. The structure is placed in a box with periodic boundary conditions. In this box not only the atoms are chemically bonded throughout 
the box, but also the atoms are bonded through the box boundaries. In this way the structure can be replicated to infinity and represents an average sample of a macroscopic structure. The size of the box $L=71 \AA$, which determined the number of $\mathrm{SiO}$ atoms necessary, was chosen based on the size of the pore which had to be carved in the structure $D=55 \AA(5.5 \mathrm{~nm})$. A cylindrical pore has been carved into the structure and the bond order was adjusted by adding hydroxyl groups to the interrupted - $\mathrm{Si}-$ bonds, and hydrogen atoms to the interrupted $-\mathrm{O}-$ bonds, thus resulting in a surface density of 1-3 hydroxyl groups per nanometer squared. The hydroxyl group density corresponded to the one observed in experiment.

\subsection{Simulations of glycerol condensed phases confined in a nanopore}

In order to simulate glycerol in a pore the previously prepared cylindrical pore in the amorphous silica must have been filled with the glycerol molecules. For this purpose earlier simulations of glycerol bulk have been used [3]. (i) In the first case a situation of a perfectly sealed pore has been simulated. The periodic bulk structure of glycerol obtained at $400 \mathrm{~K}$ has been replicated and a supercell $3 \times 3 \times 3$ with a box size of $L=120 \AA$ was obtained. The supercell contained enough glycerol molecules to fill the pore. From this glycerol structure a cylinder with dimensions corresponding to the silica pore was carved, and the cylinder was inserted into the silica pore. Then the molecular dynamics in constant number of particles, constant temperature and constant volume (NVT) ensemble and Discover molecular potential function $[7,8]$ was used to equilibrate the structure. The temperature was continuously decreased from $400 \mathrm{~K}$ down to $150 \mathrm{~K}$ with a step of $10 \mathrm{~K}$. (ii) In the second case the glycerol phase flowing into pores upon cooling was simulated. For this purpose the cylinders of glycerol condense phase have been carved from the bulk structures obtained during the previous bulk simulations at each temperature. Again the $3 \times 3 \times 3$ supercells were built while their box sizes varied from 90 to $120 \AA$. Cylinders with a constant diameter $D=55 \AA$ were carved, hence the number of glycerol molecules changed with changing density. The glycerol cylinders were inserted into the silica pores and NVT simulations at corresponding temperature were run until potential energy and cylindrical distribution functions and other simulational observables equilibrated.

\subsection{The free volume simulations}

Once the glycerol molecular structures were built and equilibrated the free volume structure and the mean cavity volumes were computed by using an algorithm developed earlier $[3,4]$. Within this algorithm the intermolecular space is probed by a probe with a so-called Bohr radius representing here the $o$-Ps probe with $R_{\mathrm{P}}=0.53 \AA$. The probe radius is experienced to provide the free volumes in good agreement with PALS measurements $[9,10]$. The probe radius is consistent also with our previous finding that sufficiently small probes should be used in order to preserve all the necessary free volume information from the computations [4]. The algorithm seeks for spaces where it is possible to insert the probe without overlaps with any of atoms. The coordinate of such the insertion is then saved. Later on the regions of mutually overlapping probes are joined together in order to recreate structure of isolated cavities. The volume and shape characteristics of the cavities are then evaluated.

\section{Results and discussion}

In order to reproduce the free volume situation of the glycerol phases confined in a silica pore, a substantial effort has been dedicated also to obtaining the realistic structure of amorphous silica. The amorphous silica gives rise to rather large free volume holes or cavities as a result of displaced $-\mathrm{SiO}_{4}$ - groups with van der Waals volume $V_{\mathrm{SiO} 4}=84 \AA^{3}$. In order to exhibit the free volume features of the amorphous silica, the free volume distribution has been calculated, as usually experimentally done in porosity measurements, as a function of probe size. The distributions were computed on a structure of pure amorphous silica and compared to that of a pure glycerol bulk (Fig. 1). In Fig. 1a it can be seen that the shape of the distribution in the pure glycerol bulk the dependence can be described by so-called exponential decay, suggesting a probabilistic distribution. In the case of the silica Fig. 1b, however, the distribution starts with a shoulder. The appearance of the shoulder infers that the cavity volumes in silica start with a jump from certain cavity volume. The existence of the shoulder appears probably due to some remnants from the crystalline silica structure. The distribution in silica decays at quite larger probe radii $R_{\mathrm{P}} \approx 2 \AA$ as compared to the pure glycerol $R_{\mathrm{P}}=1.0 \AA$.

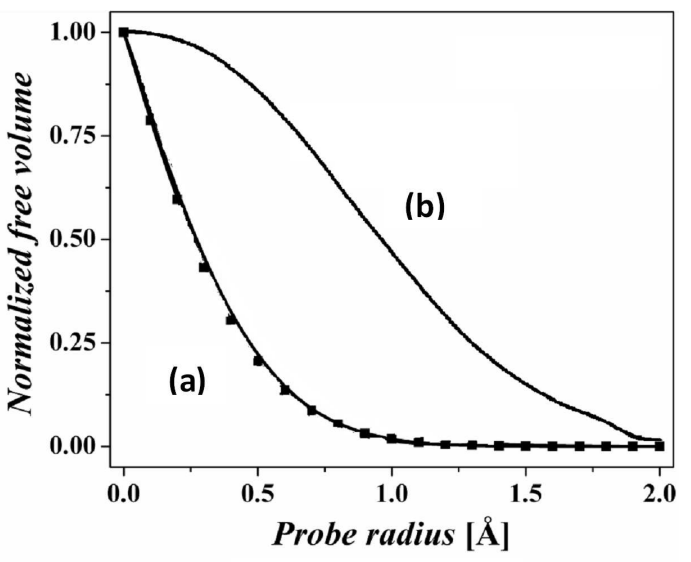

Fig. 1. The free volume distribution normalized to the so called empty space volume obtained for the point probe with a zero radius in (a) glycerol bulk and (b) amorphous silica.

Another feature of the silica pore exhibited during simulations was a rather spherical shape of the cavities as compared to the glycerol bulk. The cavity shape was evaluated in terms of a shape factor given as a sphere 
diameter with equivalent volume with a particular cavity in ratio with the longest dimension of the cavity calculated according to the algorithm presented earlier [4]. The shape factor calculated for silica reaches 0.84 versus 0.49 computed in glycerol bulk.

As the nanopore has been carved into the silica as described in Sect. 2, and the pore was filled with molecules of the glycerol condensed phase, the molecular dynamic were run until the structures equilibrated. The simulations were run for a wide range of temperatures and the free volumes were computed.

In the case of perfectly sealed pore and constant number of molecules the molecules started to get stuck to each other and adsorbe to the pore's surface as the temperature dropped and molecules started losing thermic energy to escape from the attracting forces. The affinity to the silica surface arose most likely due to the presence of the hydroxyl groups which create a complex hydrogen bonding network.

Additionally to the hydroxyls, also - $\mathrm{Si}-\mathrm{O}-\mathrm{Si}-$ bridges were present on the surface of the pore originating from the parent $-\mathrm{SiO}_{4}-$ structure. The $-\mathrm{Si}-\mathrm{O}-\mathrm{Si}-$ bridges are potentially able to take part on the hydrogen bonding due to the electronic configuration of the oxygen atom, however the formation of the hydrogen bonding was in this case suppressed by the steric hindering. Furthermore, formation of additional oxygen bridges as suggested previously [11] is supposed here to be relatively rare as compared to the concentration of $\mathrm{OH}$ groups due to the geometrical reasons. The newly formed oxygen bridges would not have an improved capability of hydrogen bonding creation hence formation of new oxygen bridges was taken completely out from consideration in the present simulations.

The hydrogen bonding between glycerol molecules caused also a mutual clustering and formation of a droplet in the middle of the pore. These features were confirmed by computed cylindrical distribution function and similar observations have been observed also on simulation on a smaller pore with $D=2.4 \mathrm{~nm} \mathrm{[5].} \mathrm{As} \mathrm{a}$ result of molecules settling down large cavities open in the middle of the pore, so creating an infinite percolating cavity in the periodic box. This leads to an increase of the cavity volume with decreasing temperature which is unprecedented observation for the bulk systems, Fig. 2a.

The second approach to the simulation of the confined glycerol phase aimed to represent a more practical case, where the glycerol phase is allowed to flow in and out upon cooling or heating. In the experimental treatment the samples of the silica pores filled with glycerol are prepared by submerging the porous glass into liquid glycerol. The glycerol phase flows in and relaxes in the pores when cooled down until freezing and it flows out and concentrates on the silica surface as the system is melted down.

For this purpose cylindrical structures of glycerol were carved from the structures obtained previously by simulated cooling of the glycerol bulk [3] for each temperature. Let us note that it is possible to obtain the equilibrium

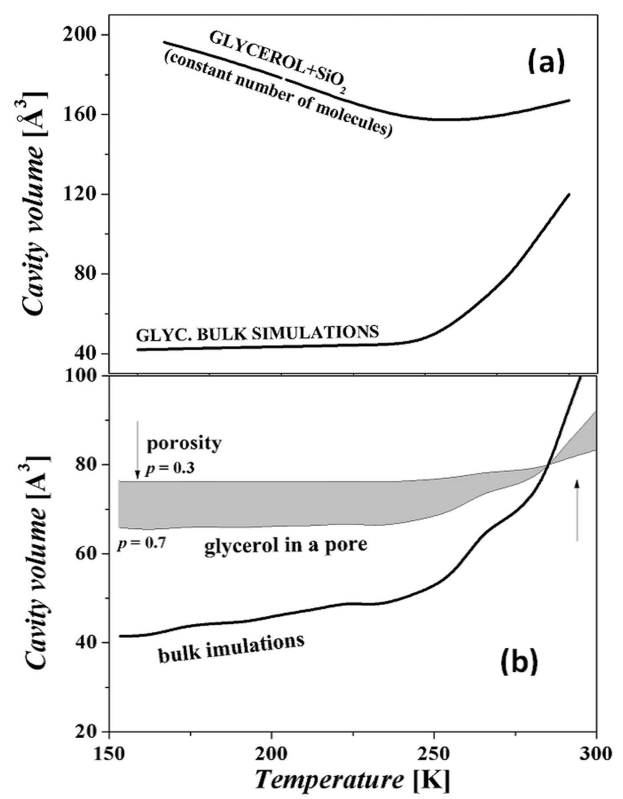

Fig. 2. The mean cavity volumes computed on the simulated glycerol condensed phases confined in a pore with $D=55 \AA$ as obtained for the case of (a) perfectly sealed pore and (b) in the pore with glycerol phase flowing in upon cooling, and compared to the previous bulk simulations. The grey area in part (b) was obtained according to Eq. (1), for different porosities considered.

molar amount of the glycerol molecules which would adsorbe to the pore at given pressure by computing adsorption isotherm based on the method developed by Frenkel et al. [12]. However this approach is very demanding on the computational power and it is time dependent, so that increased computational effort and capacity used may lead to finding a more relaxed molecular structure with higher density for a given pressure. As in this case the glycerol molecules are considerably smaller than the pore radius, it is assumed that the glycerol density in pores will be similar to that of the glycerol bulk as the confinement strength often defined as the ratio of the pore radius and molecular size [13] is rather weak.

After the structures of the glycerol were equilibrated by molecular dynamic runs in NVT ensemble, the calculation of the free volume microstructure and evaluation of the mean cavity volumes took place.

Here one has to realize that simulated pore and with the silica matrix may represent only a part of the porous network, and based on experimental data the amount of silica material versus the empty amount of pores may vary for different glasses. Because the simulations allow some generalization, we may estimate the behavior of the computed cavity volumes with temperature if the amount of the silica materials changes. The usual quantificator relating the amounts of silica and pore volumes is known as porosity parameter $p$. The mean cavity volume then given as a ratio of the free volume contained in the silica and glycerol, divided by the corresponding numbers of 
cavities

$$
\bar{V}_{\mathrm{h}}=\frac{(1-p) f^{\mathrm{Si}}+p f^{\mathrm{GLY}}(T)}{(1-p) f^{\mathrm{Si}} / \bar{V}_{\mathrm{h}}^{\mathrm{Si}}+p f^{\mathrm{GLY}}(T) / \bar{V}_{\mathrm{h}}^{\mathrm{GLY}}(T)},
$$

where $V_{\mathrm{h}}$ is the mean cavity volume, $p$ - porosity, $f$ is the free volume fraction and Si and GLY stand for a particular material, silica and glycerol, respectively. Variable $T$ is temperature, as in the glycerol the free volume fraction is temperature dependent; while silica has the coefficient of thermal expansion $10^{3}$ times lower than glycerol and can be held as constant. Usual porosities for CPG glasses determined experimentally vary between $0.5-0.7$ [14]. The resulting picture on the mean cavity volume dependence with temperature is shown in Fig. $2 \mathrm{~b}$ as a grey area obtained for porosities between $p=0.3-0.7$.

The computed dependence is in a good agreement with the picture obtained from experimental measurements of mean free hole volumes by PALS in a pore [2]. The dependence is more flat as compared to the glycerol bulk, while the indicated amounts of the mean cavity sizes are about twice larger consistently with the experiment. The computed temperature dependence of the cavity volumes in a pore crosses with the line computed for the bulk at higher temperatures, at around $290 \mathrm{~K}$ (Fig. 2b). This is perhaps where the mean cavity volume of the pure silica matrix equals to the mean cavity volume in glycerol. The similar crossing can be observed on the experimental data, too [2], however there is only one point of experimental data in that region before the data saturate into the plateau region.

Existing PALS experimental works have shown that the onset of the plateau can be shifted in confinement to both lower and higher temperatures. In the high temperature region the positronium enters the regime where it acts more like a digger and may increase the size of the free volume holes. The size to which the o-Ps pushes apart the cavity walls may be decreased by the effect of confinement. This may be responsible for the apparent shift of the onset of the plateau region towards lower temperatures in some substances.

On the other hand, it has been also shown that the temperature region, where the regime of the $o$-Ps existence changes, can be distinguished by a change of dynamic picture on the structure within a time window corresponding to the lifetime of $o-\mathrm{Ps}[15]$; a well defined cavity structure at higher temperatures lasts shorter than a lifetime of $o$-Ps atom, as the dynamics and translocation of molecular bodies become faster. It is known that the presence of the confinement walls slows down the dynamics and diffusion of molecules. This will be eminent particularly in the case of confinement walls with strong intermolecular forces. Slowing down the dynamics in the structure might shift the onset of the plateau towards higher temperatures.

\section{Conclusions}

Within the present work the free volume of glycerol condensed phases in confinement has been explored by means of computer simulations. The computations infer that the aspect of filling will be important for the experimentalists. Two cases of the glycerol confined phases were considered.

Firstly, the situation of a perfectly sealed pore shows that large cavities may open upon cooling as molecules adsorb to the pore surface and cluster together. As a result the mean cavity volume may rise with decreasing temperature what is a picture unprecedented in observations by PALS in the bulk of pure substances.

Secondly, when a "working" phase of glycerol is considered, when the glycerol molecules are allowed to flow in and out during cooling, or heating respectively, the computed mean cavity volume dependence with temperature shows a more flat behavior, with about doubled cavity volumes as compared to the bulk. This picture well agrees with experimental PALS measurements. The computations infer that the mean cavity volume and its temperature dependence are in greater extent affected by the free volume holes in the silica material which forms the porous structure.

\section{Acknowledgments}

This work was supported by Slovak Research and Development Agency (grant No. SRDA-0451-11), and Scientific grant agency of the Ministry of Education of the Slovak Republic (VEGA grant Nos. 2/0093/12 and $2 / 0068 / 13)$.

\section{References}

[1] G.B. McKenna, Eur. Phys. J. Special Topics 189, 285 (2010).

[2] D. Kilburn, E.P. Sokol, V.G. Sakai, A.M. Alam, Appl. Phys. Lett. 92, 033109 (2008).

[3] D. Račko, R. Chelli, G. Cardini, S. Califano, J. Bartos, Eur. Phys. J. D 32, 289 (2005).

[4] D. Račko, S. Capponi, F. Alvarez, J. Colmenero, J. Chem. Phys. 134, 044512 (2011).

[5] R. Busselez, R. Lefort, Q. Ji, F. Affouard, D. Morineau, Phys. Chem. Chem. Phys. 11, 11127 (2009).

[6] R. Vink, G. Barkema, Phys. Rev. B 67, 245201 (2003).

[7] S.W. Bunte, H. Sun, J. Phys. Chem. B 104, 2477 (2000).

[8] H. Sun, J. Phys. Chem. B 102, 7338 (1998).

[9] S. Arizzi, P.H. Mott, U.W. Suter, J. Polym. Sci., Part B, Polym. Phys. 30, 415 (1992).

[10] J.-E. Kluin, T. Yu, S. Vleeshouwers, J.D. McGervey, A.M. Jamieson, R. Simha, K. Sommeril, Macromolecules 26, 1853 (1993).

[11] L.T. Zhuravlev, Colloids Surf. A 173, 1 (2000).

[12] J.I. Siepmann, D. Frenkel, Mol. Phys. 75, 59 (1992).

[13] D. Račko, P. Cifra, J. Chem. Phys. 138, 184904 (2013).

[14] O. Šolcová, et al., Appl. Catal. A General 313, 167 (2006).

[15] D. Račko, J. Krištiak, Mater. Sci. Forum 733, 33 (2013). 